\title{
Efficacy of Combined Therapy with Nitroimidazole Drugs and Broad-Spectrum Antibiotics on Intra- Abdominal Infection: A 10 Years Real-World Cohort Study
}

\section{Hongyi Liang}

General Hospital of Southern Theatre Command of PLA

\section{Gaici Xue}

General Hospital of Southern Theatre Command of PLA

Jiasheng Ye

General Hospital of Southern Theatre Command of PLA

Fangfang Wen

General Hospital of Southern Theatre Command of PLA

\section{Leifang Ouyang}

General Hospital of Southern Theatre Command of PLA

Jingjing Ji

General Hospital of Southern Theatre Command of PLA

Bo Ji

General Hospital of Southern Theatre Command of PLA

Zhifeng Liu ( $\square$ Zhifengliu7797@163.com )

General Hospital of Southern Theatre Command of PLA

\section{Research Article}

Keywords: Abdominal infection, Broad-spectrum antibiotic, Nitroimidazoles, Clinical efficacy

Posted Date: January 10th, 2022

DOI: https://doi.org/10.21203/rs.3.rs-1042948/v2

License: (c) (i) This work is licensed under a Creative Commons Attribution 4.0 International License. Read Full License 


\section{Abstract}

Background: Abdominal infection still has a high mortality rate, and whether the combination of nitroimidazoles against obligate anaerobe could improve the outcome of abdominal infection remains controversial.

Objective: This study aimed at exploring the clinical efficacy of broad-spectrum antibiotics combined with nitroimidazoles in the treatment of abdominal infection.

Methods: Clinical data of patients with abdominal infection who received broad-spectrum antibiotics alone (BSA) or broad-spectrum antibiotics in combination with nitroimidazole (NCBSA) at a single center from June 2010 to May 2020 were retrospectively reviewed. One-to-one propensity score matching (PSM) was performed to adjust the potential differences in age, gender, APACHE II score and SOFA score of the two groups. Clinical effective rate, hospitalization days and total hospitalization costs were compared between the two groups. Meanwhile, subgroup efficacy was analyzed according to disease severity (APACH II score) and whether abdominal surgery was performed.

Result: A total of 149 propensity score-matched case pairs were included in this study. There was no statistical difference in clinical effective rates between the two groups $(P=0.236)$. The patients in BSA group had shorter hospital stays (18d vs $27 \mathrm{~d}, P=0.001$ ) and lower hospitalization costs (85185.7 yuan vs 187898.3 yuan, $P<0.001)$. However, the body temperature and PCT in patients in NCBSA group were higher than those in BSA group ( $P=0.007$ and $P=0.045$, respectively), and CRP and WBC values in NCBSA group also were higher, but there were no significant differences between the two groups $(P=$ 0.105 and $P=0.170$, respectively). And more patients (92 vs 38) in NCBSA group underwent abdominal surgery, which indicated the infection and clinical conditons in NCBSA group were probably more severe. Subgroup analysis showed that the severity of the disease and whether the patient suffered postoperative co-infection did not affect the therapeutic effect of the two groups $(P>0.05)$.

Conclusion: The clinical efficacy of broad-spectrum antibiotics combined with nitroimidazoles for abdominal infection was similar to that of broad-spectrum antibiotics alone, and whether the combination of nitroimidazoles in these conditions still needs more lcinical evidence.

\section{Introduction}

Abdominal infection is a common disease and complication of abdominal surgery. As the second most common infectious disease in inpatients ${ }^{[1]}$, the case fatality rate of patients with abdominal infection reaches $20 \%{ }^{[2]}$, which is a major cause of non-traumatic death in inpatients.

Abdominal infection involves a wide range of gut microbes, including the Enterobacteriaceae (e.g., Klebsiella pneumoniae, Escherichia coli), enterococcus, streptococcus, and Bacteroides (especially Bacteroides fragility $)^{[3-4]}$. Although the number of Bacteroides fragilis only accounts for $1 \%$ of the colonic microflora, it accounts for $60 \%-90 \%$ of all anaerobic bacterial infections, and is the main anaerobic 
pathogenic bacteria leading to abdominal infection and sepsis ${ }^{[5]}$. Early fluid resuscitation, dysfunctional organ support, infection source control and rational use of antibiotics are important means for the treatment of abdominal infection, among which infection source control is the core link ${ }^{[4,6-9]}$. During effective infection source control, anti-infective therapy should be given as soon as possible, and antianaerobic drug therapy should be accompanied by the whole course of antibiotics ${ }^{[10]}$. The recommended drugs for the treatment of abdominal infection are similar in the guidelines ${ }^{[2-4,11]}$. Broad-spectrum antibiotics that cover anaerobic bacteria in combination with nitroimidazoles are generally not recommended regardless of the complexity of abdominal infection. However, in the context of increasing multidrug-resistant bacteria in the world, the effect of simple empirical use of broad-spectrum antibiotics to control abdominal infection is not idealistic. Especially in recent years, the resistance of Bacteroides fragile to clindamycin, cefoxitin, moxifloxacin, meropenem, ertapenem, imipenem,

piperacillin/tazobactam and other anti-anaerobic drugs has increased significantly ${ }^{[12-14]}$. Therefore, some scholars suggest that nitroimidazoles against obligate anaerobes should be used in addition to broad-

spectrum antibiotics for the treatment of abdominal infection to improve the infection control rate ${ }^{[15]}$. We have also found in practical clinical work that in some cases of abdominal infection, although piperacillin-tazobactam or imipenem and cilastatin, meropenem, etc. which can be covered with anaerobes have been used for initial empirical treatment, treatment results are often unsatisfactory, but the addition of nitroimidazoles as anti-anaerobes seems to improve the patient's condition. This paper was to investigate the clinical efficacy of broad-spectrum antibiotics combined with nitroimidazoles for abdominal infection. Firstly, we retrospectively analyzed the cases of abdominal infection at our center in the past 10 years and designed a propensity score matching (PSM) cohort study, then compared the clinical efficacy between broad-spectrum antibiotics (BSA) alone and broad-spectrum antibiotics combined with nitroimidazoles (NCBSA) against obligate anaerobes in the treatment of abdominal infection to further evaluate whether broad-spectrum antibiotics combined with nitroimidazoles against obligate anaerobes could improve the control rate of abdominal infection.

\section{Materials And Methods}

\section{Patient selection}

The subjects in this study included patients from a high-volume center in China who received intraabdominal infection during hospitalization from June 1, 2010 to May 31, 2020. Inclusion criteria: adult patients aged 18 years and above with uncomplicated and complex abdominal infection, that is, abdominal pain or systemic inflammatory reaction, and the infection is confined to the gastrointestinal wall or penetrates the abdominal cavity organs and enters the peritoneum, accompanied by peritonitis or abdominal abscess formation ${ }^{[10]}$. Exclusion criteria: (1) patients with advanced tumor, (2) patients receiving nitroimidazoles by non-intravenous means, and (3) patients with only nitroimidazoles in the anti-infective regimen.

\section{Study design}


This study was a retrospective analysis comparing the efficacy of nitroimidazole drugs combined with broad-spectrum antibiotics (NCBSA) and broad-spectrum antibiotics (BSA) on intra-abdominal infection in the real world. We collected each patient's ID, gender, age, diagnosis, time of admission/discharge, Acute Physiology and Chronic Health Evaluation (APACHE) $₫$ score, Sequential Organ Failure Assessment (SOFA) score, nitroimidazole drugs and broad-spectrum antibiotics dosage, start and stop time, drug administration route, infection index[body temperature, routine blood parameters(such as white blood cell, WBC), C-reactive protein(CRP) and procalcitonin (PCT)levels], liver and kidney function results, bacterial Gram stain and culture test results, and all expenses during hospitalization. Propensity scorematched (PSM) factors included age, gender, APACHE $\$ score and SOFA score.

In this retrospective cohort study, the consistency of the efficacy between the two groups was evaluated by the clinical efficiency and microbial success rate after PSM. The standards of clinical efficiency were promulgated by the Ministry of Health, with categories including cured, markedly effective, progressive, and ineffective. 'Cured' indicates that a patient's symptoms, signs, and abnormal laboratory results were resolved, and the offending bacteria were completely eradicated. 'Markedly effective' indicates that the condition of a patient was substantially improved, but one of the symptoms, signs, laboratory test results or bacteriology results remained abnormal. 'Progressive' indicates that the condition of a patient was improved, but one or more of the symptoms, signs, laboratory test results, or bacteriology results have not returned to normal. 'Ineffective' indicates that after $72 \mathrm{~h}$ of treatment, the condition of a patient did not improve in terms of symptoms, signs, laboratory tests, or bacteriology assessments. Markedly effective responses are indicative of successful treatment and contributed to the clinical efficiency calculation.

The study protocol was reviewed and approved by the Ethics Committee of the General Hospital of the Southern Theatre Command. Written informed consent for participation was not required for this study in accordance with the national legislation and the institutional requirements.

\section{Statistical analysis}

Statistical analysis was performed using IBM SPSS software (version 22.0, Chicago, IL, USA). 1:1 PSM was performed with a logistic regression model to adjust for potential biases in age, gender, APACHE $\square$ score and SOFA score between the two groups. Nearest neighbor matching and non-replacement sampling were used with a caliper distance of 0.2 and matching order of largest. And, Pearson chi-square test, Fisher exact test, independent samples t-test, or nonparametric test was used for statistical analysis as appropriate. Categorical variables were presented as frequency, and continuous variables were presented as mean \pm standard deviation ( $\pm s$ ) or median (the first quartile, the third quartile) as appropriate. A $P$ value $<0.05$ was considered statistically significant.

\section{Results}

\section{Patient inclusion results}


In 10 years, 479 patients were diagnosed with intraperitoneal infection (including intestinal fistula), excluding 62 patients who didn't meet the definition of intraperitoneal infection ${ }^{[10]}, 23$ patients who were treated with nitroimidazoles by non-intravenous means, and 39 patients who were treated with nitroimidazoles alone, leaving 355 valid data. Among them, 158 patients were treated with broadspectrum antibiotics combined with nitroimidazoles and 197 patients with broad-spectrum antibiotics alone. A total of 298 patients were included after 1:1 PSM (Figure 1).

\section{Patients' baseline characteristics}

As shown in Table 1, compared with the BSA group, the patients in the NCBSA group were younger with $54.1 \pm 17.7$ years vs $60.0 \pm 19.3$ years $(P=0.004)$, but gender, APACHE II score $(P=0.433)$ and SOFA score $(P=0.634)$ were generally balanced.

According to the sample numbers of the two groups, the current study used 1:1 PSM, there are 149 patients in each group. After matching, a total of 57 samples were screened out. The baseline data and $P$ values after matching the two sets of data were shown in Table 1 . The $P$-value of all variables after matching was greater than 0.05 (Table 1). Covariate balance was massively improved in the matched sample compared to original sample (Figure S1 and Table S1)

\section{The clinical outcomes of the two groups}

The anti-infection regimens of the single-use group treated with piperacillin-tazobactam/cefoperazone sulbactam in 38 cases, imipenem and cilastatin sodium/meropenem/ertapenem in 36 cases, cefuroxime/ceftriaxone/ceftazidime/ cefazolin/ in 33 cases, tegacycline in 3 cases, doxycycline in 2 cases, gentamicin/moxifloxacin in 37 cases. Combination group anti-infection regimen using piperacillintazobactam/cefoperazone sulbactam were in 52 cases, Imipenem and cilastatin sodium/Meropenem/ertapenem in 42 cases, cefazolin/cefuroxime/cefoxitin /ceftriaxone/ceftazidime in 46 cases, tegacycline in 2 cases, ciprofloxacin/moxifloxacin/vancomycin/teicoplanin/cappofenjing in 7 cases. All patients were treated with metronidazole/ornidazole/tinidazole/leornidazole.

Before matching and after matching (Table 2), the clinical response comparison between the two groups showed no significant difference in the clinical cure rate (before matching: $83.8 \%$ versus $78.1 \%, P=0.261$, after matching: $83.9 \%$ versus $78.5 \%, P=0.236$ ). The median total length of hospitalization in BSA group was 9 days less than that in NCBSA group, statistically significant $(P=0.001)$. According to the infection indicators (Table 3), the body temperature of the BSA group after matching and the PCT were lower than those in NCBSA group ( $P=0.007$ and $P=0.045$, respectively). Although CRP and WBC were alsoo lower in BSA group than those inNCBSA group, there were no statistical differences $(P=0.105$ and $P=0.170$, respectively), which indicated the infection in NCBSA group was more serious. In terms of the severity of patients' illness, no matter how much APACHE II score was scored, there was no statistical difference in efficacy between the two groups $(P>0.05)$ (Table 4). However, it should be noted that the effective rate of the NCBSA group was higher than that of the BSA group (76.5\% versus $65.0 \%)$ on APACHE II score of 11 15. However, because of the small number of cases, there was no significant difference between the two 
groups $(P>0.05)$. Surgery or not did not affect the overall efficacy of the two groups $(P>0.05)$, but the effective rate of patients undergoing surgery in the BSA group was higher than that in the NCBSA group $(94.7 \%$ vs $79.3 \%, P<0.05)$ (Table 5). In BSA group, 38 patients underwent abdominal surgery, and the median hospitalization days was 18.5 days. 111 patients underwent non-abdominal surgery, and the median hospitalization days was 18.0 days. In NCBSA group, 92 patients underwent abdominal surgery, and the median hospitalization days was 33.5 days. 57 patients underwent non-abdominal surgery, and the median hospitalization days was 17.0 days (Table 6).

\section{Economical efficiency}

The median total hospitalization cost of the BSA group was nearly 100,000 yuan lower than that of the NCBSA group, and there was a statistical difference between the two groups $₫ P \otimes 0.001$ \\Table $2 \rrbracket$. Subgroup analysis showed that the median cost of abdominal surgery in BSA group was 117648.1yuan, and the median cost of abdominal surgery in NCBSA group was 219313.9 yuan (Table 7).

\section{Safety profile}

As this study is a retrospective study, the adverse reactions of patients can only be extracted from the adverse reactions supervision system. After investigation, no adverse reactions were reported by all patients in this study.

\section{Discussion}

In this study, the PSM method was used to compare the clinical efficacy of broad-spectrum antibiotics and broad-spectrum antibiotics combined with nitroimidazoles in the treatment of abdominal infection. The results showed that although the basic condition matching was consistent, the infection index and the surgical patients of a combination of broad-spectrum antibiotics with nitroimidazoles were higher than that in the group of broad-spectrum antibiotics. The clinical effective rate of broad-spectrum antibiotics combined with nitroimidazole in the treatment of abdominal infection was similar to that of broad-spectrum antibiotics, while the hospitalization days of patients in the combination group were longer and the cost of hospitalization was higher.

This study was the first to directly compare the efficacy of broad-spectrum antibiotics and broadspectrum antibiotics combined with nitroimidazoles in the treatment of abdominal infection. The results showed that there was no significant difference in the clinical effective rate between the NCBSA and BSA in treatment of abdominal infection, which was mainly consistent with the recommendations of previous studies and current guidelines ${ }^{[2-4,11]}$. As some scholars have shown, NCBSA can not improve the therapeutic effect of perforated appendicitis ${ }^{[17]}$, nor can it reduce the deterioration rate of necrotizing enterocolitis ${ }^{[18]}$. Solomkin et al. performed a randomized trial to assess the safety and efficacy of Ceftolozane/Tazobactam plus metronidazole in intra-abdominal infections. And the results indicated that this combination regimen was non-inferior to meropenem for patients with complicated intra-abdominal infections ${ }^{[19]}$. Similarly, another study from Qin et al. demonstrated that ceftazidime/avibactam in 
combination with metronidazole was not inferior to meropenem in the treatment of complicated intraabdominal infections in $\mathrm{Asia}^{[20]}$.

Combining our results for further analysis, there may be three reasons for the no difference in the effectiveness of the two groups. First of all, the detection rate of carbapenemase resistance genes in Enterobacteriaceae isolated from abdominal infections is increasing year by year ${ }^{[21]}$, which increases the difficulty of treating abdominal infections. Second, in the selection of nitroimidazole drugs, the drugs used in the combination group in this study were 23 cases of metronidazole, 119 cases of ornidazole, and 7 cases of levornidazole. Among them, metronidazole is the first-generation nitroimidazole drug, ornidazole is the third-generation nitroimidazole drug, and levornidazole is the levorotatory isomer of the third-generation ornidazole. Although there was a lack of clear drug resistance data, second and thirdgeneration nitroimidazole antibiotics (such as Levoornidazole) are significantly better than metronidazole in the treatment of abdominal anaerobic infections ${ }^{[22]}$. Studies have shown that compared with ornidazole, levornidazole has obvious advantages in the treatment of anaerobic infections ${ }^{[23-24]}$, and the clinical effect of treating abdominal infections is better than ornidazole ${ }^{[24]}$. However, due to the small number of levornidazole used in the combination group, the efficacy of combined nitroimidazole drugs may not be reflected. Finally, the overall span of this research is 10 years, during which many treatment concepts and technologies are continuously improved. The difference in the treatment effect in different periods, in particular, the low treatment effect caused by the backward treatment technology in the early stage may be one of the reasons for the comprehensive indifference.

However, in this study, we also found that after PSM the basic clinical features, the infection indexes such as PCT and Temperature in the combination group were significantly higher than those in BSA group, and although there was no statistical difference, WBC and CRP were also higher than those in BSA group, which may suggest that the infection in the combination group was more severe. Besides, more patients (92 vs 38) in NCBSA group underwent abdominal surgery, which was also in line with the doctor's habit of an empirical combination of drugs, that is, it seems that the infection or the clinical conditions are more severe and tends to be combined with drugs. And this may be one of the reasons why the combination group had longer hospitalization days and higher costs in this study. Therefore, we could also speculate that under the premise of more severe infection and clinical conditions in the combination group, the curative effect of the two groups is the same, and on the other hand, the efficiency of the combination group may be higher. However, due to the complexity of abdominal infections, such as the source of infection, the treatment of primary infections and other clinical data complexity, we cannot fully balance the severity of all infections and clinical conditions for the time being, and we have not continuously dynamically assessed changes in infection indicators, therefore, we still can not conclude that the combination of drugs is more effective. This is also the deficiency of this study, which needs to be confirmed by more prospective studies in the future.

In addition, a subgroup analysis of abdominal surgery found that the effective rate of patients with abdominal surgery in the single-use group was higher than that in the combination group, but because we 
did not record the length of the operation and the amount of blood loss, we could not match the size of the surgery and the severity of the postoperative condition of the two groups. Therefore, the results may be biased. However, it is worth noting that in the subgroup analysis of the severity of the disease, we found that although there was no statistical difference in the severity of the disease for empirical treatment of abdominal infection alone or in combination, when the APACHEll score was 11-15, the effective rate of broad-spectrum antibiotics combined with nitroimidazoles was higher than that of broadspectrum antibiotics alone (76.5\% versus $65.0 \%$ ), but the difference was not statistically significant. This may be because neither too severe nor too mild can reflect the advantage of the combination group, but just when the severity of the disease is moderate can reflect the advantage of the combination therapy. In future studies, as the number of cases increases, the advantages of broad-spectrum antibiotics combined with nitroimidazole drugs in the treatment of abdominal infections under this condition are likely to be revealed.

Finally, we also compared the safety of the two groups, and our results showed that there were no more obvious side effects in the combination group. More recently, a meta-analysis repotted by Che et al showed that there were no significant differences in overall adverse events, severe adverse events or mortality between $\beta$-lactam/ $\beta$-lactamase inhibitors combined with metronidazole and carbapenem in the treatment of complex abdominal infection, but the risk of vomiting was higher in the combination group $^{[25]}$. This is consistent with our findings.

In this study, there are also some limitations. First, it is a retrospective single-center study with relatively small sample size. In addition, this study spans a long period of 10 years, and the difference in effect caused by the progress of treatment technology may affect the results of the study. Future prospective, multi-center, large sample research is expected to provide us with more reliable evidence.

\section{Conclusion}

The clinical efficacy of broad-spectrum antibiotics combined with nitroimidazoles for abdominal infection was similar to that of broad-spectrum antibiotics alone, How to choose combination therapy to improve the treatment efficiency of abdominal infection still needs prospective, multi-center, large sample studies in the future to provide more reliable evidence.

\section{Abbreviations}

NCBSA: Nitroimidazole Drugs Combined With Broad-Spectrum Antibiotics

BSA: Broad-Spectrum Antibiotics

PSM: Propensity Score Matching

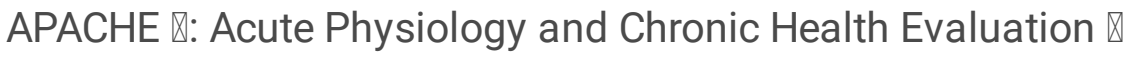


SOFA: Sequential Organ Failure Assessment

WBC: White Blood Cell

CRP: C-reactive protein

PCT: Procalcitonin

\section{Declarations}

\section{Availability of supporting data}

The datasets used and/or analyzed during the current study are available from the corresponding author on reasonable request.

\section{Acknowledgments}

None.

\section{Fundings}

This work was supported by grants from the National Natural Science Foundation of China [N0.82072143].

\section{Authors' controbutions}

Study design, literature research, manuscript editing, Hongyi Liang and Gaici Xue, data acquisition: Jiasheng Ye, statistical analysis: Gaici Xue, revised and polished the manuscript: Fangfang Wen, Leifang Ouyang, Jingjing Ji, Study design, literature research, manuscript editing, manuscript final version approval: Zhifeng Liu and Bo Ji.

\section{Competing interests}

The authors of this work declare that they have no potential conflicts of interest, including relevant financial interests, activities, relationships and affiliations.

\section{Ethics approval}

A retrospective review and an analysis of medical records were conducted. The confidentiality of data was maintained. The studies involving human participants were reviewed and approved by the Ethics Committee of the General Hospital of the Southern Theater Command. Written informed consent for participation was not required for this study in accordance with the national legislation and the institutional requirements.

\section{Consent for publication}


Not applicable.

\section{References}

1. Vincent JL, Rello J, Marshall J, et al. International study of the prevalence and outcomes of infection in intensive care units. JAMA, 2009, 302(21): 2323-2329.

2. Chinese Society of Surgical Infection and Intensive Care, Chinese Society of Surgery, Chinese Medical Association, Chinese College of Gastrointestinal Fistula Surgeons, Chinese College of Surgeons, Chinese Medical Doctor Association.Chinese guideline for the diagnosis and management of intra- abdominal infection (2019 edition). Chinese Journal of Practical Surgery, 2019, 40(1): 1-16.

3. John E. Mazuski, Jeffrey M.et al. The Surgical Infection Society Revised Guidelines on the Management of Intra-Abdominal Infection[J]. SURGICAL INFECTIONS.2017,18(1).

4. Sartelli M, Chichom-Mefire A, Labricciosa FM, et al. The management of intra-abdominal infections from a global perspective: 2017 WSES guidelines for management of intraabdominal infections. World Journal of Emergency Surgery (2017) 12:29.

5. Wang Yanyan, Han Yanqiu, Guo Sufang, et al.Analysis on the distribution and drug resistance features of anaerobes isolated from clinical infectious specimens[J].National Medical Journal of China, 2018, 96 (36):2894-2898.

6. Marshall JC, Maier RV, Jimenez M, et al. Source control in the management of severe sepsis and septic shock: an evidence-based review[J].Crit Care Med,2004,32(11 Suppl):S513-526.

7. Tellor B, Skrupky LP, Symons W, et al. Inadequate source control and inappropriate antibiotics are key

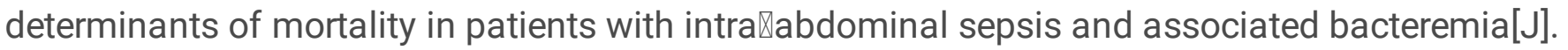
Surg Infect(Larchmt),2015,16(6):785-793.

8. van de Groep K, Verhoeff TL, Verboom DM, et al. Epidemiology and outcomes of source control procedures in critically ill patients with intra-abdominal infection[J]. J Crit Care. 2019,52:258-264.

9. Lalisang TJM, Usman N, Hendrawidjaya I, et al. Clinical Practice Guidelines in Complicated IntraAbdominal Infection 2018: An Indonesian Perspective[J]. Surg Infect (Larchmt). $2019,20(1): 83-90$.

10. Stijn Blot, Jan J. Essentials for selecting antimicrobia in therapy for intra-abdominal infections. Therapy in practice, 2012, 72(6): e17-e32.

11. Solomkin JS, Mazuski JE, Bradley JS, et al. Diagnosis and management of complicated intraabdominal infection in adults and children: guidelines by the Surgical Infection Society and the Infectious Diseases Society of America. Clin Infect Dis, 2010, 50(2):133-164.

12. Hecht DW. Prevalence of antibiotic resistance in anaerobic bacteria $\varangle$ worrisome developments[J].Clin Infect Dis $₫ 2004,39(1): 92 \varangle 97$.

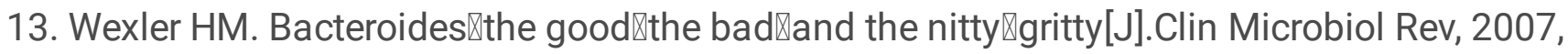
20(4):593ه621.

14. Yao Wang, Xin-Fei Chen, Ying-chun Xu, et al. Evaluation of VITEK MS, Clin-ToF-II MS, Autof MS 1000 and VITEK 2 ANC card for identification of Bacteroides fragilis group isolates and antimicrobial 
susceptibilities of these isolates in a Chinese university hospital[J]. Microbiology, Immunology and Infection, 2019,52(3):456-464.

15. Gao SM, ZHANG YS.Analysis of the efficacy of piperacillin/ tazobactam combined with metronidazole in the treatment of abdominal infection[J]. Contemporary Medical Symposium,2020,18(3):142-143.

16. Lan Haigou, Li Yanjuan, WANG Nandou, et al. The effect of piperacillin/tazobactam combined with metronidazole on patients with severe abdominal infection and its effect on the improvement of inflammatory factors [J]. Anti Infect Pharm 2019 August, 16(8):1465-1467.

17. Shang Qingjuan GengQiankun, Zhang Xuebing, et al. The efficacy of combined therapy with metronidazole and broad-spectrum antibiotics on postoperative outcomes for pediatric patients with perforated appendicitis[J]. Medicine (2017) 96:47.

18. LUO Lijuan. Broad-spectrum antibiotics plus metronidazole may not prevent the deterioration of

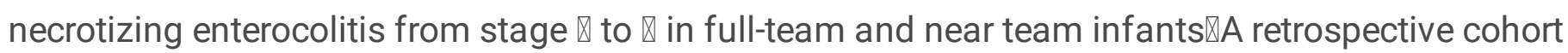
study[D]. Chongqing Medical University,2015.

19. Mazuski JE, Gasink LB, Armstrong J, et al. Efficacy and Safety of Ceftazidime-Avibactam Plus Metronidazole Versus Meropenem in the Treatment of Complicated Intra-abdominal Infection: Results From a Randomized, Controlled, Double-Blind, Phase 3 Program. Clin Infect Dis. 2016, 62(11):1380-1389..

20. Qin X, Tran BG, Kim MJ, et al. A randomised, double-blind, phase 3 study comparing the efficacy and safety of ceftazidime/avibactam plus metronidazole versus meropenem for complicated intraabdominal infections in hospitalised adults in Asia. Int J Antimicrob Agents. 2017, 49 (5):579-588..

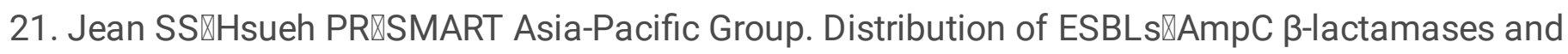
carbapenemases among Enterobacteriaceae isolates causing intra-abdominal and urinary tract infections in the Asia-Pacific region during 2008-14『results from the Study for Monitoring Antimicrobial Resistance Trends $\triangle$ SMART囚. J Antimicrob Chemother $\ 2017 \otimes 72(1) \otimes 166-171$.

22. XIE Shengxin, WANG Zhiqiang, GU Yanyan, et al. Clinical study of Levornidazole Sodium Chloride Injections in the treatment of oral, pelvic and abdominal anaerobic infection[J]. Northwest Pharmaceutical Journal, 2019, 34(05):677-680.

23. Li Weiwei囚Yao Minna囚Zhao Xian, et al. Systematic \eview of Levornidazole in the Treatment of Anaerobic InfectionChinese Journal of Pharmacoepidemiology[J]. 2019, 28(03): 151 -157.

24. ZHANG Dezhi, LIU Meng, ZHU Shaogong,et al. Effect of Levoornidazole and Sodium Chloride Injection in the Treatment of Abdominal Anaerobic Bacteria Infection[J]. Henan Journal of Surgery,2019,06:60-62.

25. Che H, Wang J, Wang R, et al. Novel Beta-Lactam/Beta-Lactamase Plus Metronidazole vs Carbapenem for Complicated Intra-abdominal Infections: A Meta-analysis of Randomized Controlled Trials. Open Forum Infect Dis. 2020, 8(1):ofaa591.

\section{Tables}


Table 1 Baseline data and balance of patients before and after propensity score matching

\begin{tabular}{lllllll} 
& \multicolumn{2}{l}{ Before matching } & \multicolumn{3}{l}{ After matching } \\
\hline General Information & BSA & NCBSA & P & BSA & NCBSA & P \\
\hline $\mathrm{n}$ & 197 & 158 & & 149 & 149 & \\
\hline Age, years & $60.0 \pm 19.3$ & $54.1 \pm 17.7$ & 0.004 & $55.7 \pm 16.8$ & $54.4 \pm 17.2$ & 0.514 \\
\hline Female & $47(23.9)$ & $44(27.8)$ & 0.392 & $42(28.2)$ & $39(26.2)$ & 0.696 \\
\hline APACHE II score & $7.0(5.0-10.0)$ & $7.0(4.0-9.5)$ & 0.433 & $7.0(5.0-10.0)$ & $7.0(5.0-9.5)$ & 0.961 \\
\hline SOFA score & $1.0(0.0-3.0)$ & $1.0(0.0-3.0)$ & 0.634 & $1.0(0.0-2.0)$ & $1.0(0.0-3.0)$ & 0.952
\end{tabular}

SOFA: Sepsis-related Organ Failure Assessment; APACHE II: Acute Physiology and Chronic Health Enquiry; BSA: broad-spectrum antibiotics; NCBSA: nitroimidazoles combined with broad-spectrum antibiotics

Table 2 Differences in effective rates, hospitalization days, and expenses between the two groups before and after propensity score matching

\begin{tabular}{lllllll} 
& \multicolumn{1}{l}{ Before matching } & \multicolumn{3}{l}{ After matching } \\
\hline General Information & BSA & NCBSA & P & BSA & NCBSA & P \\
& 197 & 158 & & 149 & 149 & \\
\hline Prognosis & & & & & & \\
\hline Effective & $165(83.8 \%)$ & $125(78.1 \%)$ & 0.261 & $125(83.9 \%)$ & $117(78.5 \%)$ & 0.236 \\
\hline Invalid & $32(16.2 \%)$ & $33(20.9 \%)$ & 0.068 & $24(16.1 \%)$ & $32(21.5 \%)$ & 0.105 \\
\hline $\begin{array}{l}\text { Hospitalization } \\
\text { expenses(yuan) }\end{array}$ & 93811.0 & 179798.0 & 0.000 & 85185.7 & 187898.3 & 0.000 \\
& $(33688.0-$ & $(73965.9-$ & & $(24028.0-$ & $(75843.0-$ & \\
\hline $\begin{array}{l}\text { Hospital } \\
\text { stays(days) }\end{array}$ & $203734.3)$ & $386154.9)$ & & $313174.3)$ & $374426.3)$ & \\
\hline $2.0(11-46)$ & $27.0(15-48)$ & 0.144 & $18.0(10-34)$ & $27.0(15-47)$ & 0.001
\end{tabular}

BSA: broad-spectrum antibiotics; NCBSA: nitroimidazoles combined with broad-spectrum antibiotics

Table 3 Infection index results between the two groups after propensity score matching 


\begin{tabular}{llll} 
Infection index & BSA & NCBSA & $\mathrm{P}$ \\
\hline $\mathrm{n}$ & 149 & 149 & \\
\hline Temperature $\left({ }^{\circ} \mathrm{C}\right)$ & $36.6(36.4-37.0)$ & $36.9(36.5-37.5)$ & 0.007 \\
\hline Procalcitonin(ug/L) & $1.0(0.41-2.7)$ & $2.2(0.6-4.9)$ & 0.045 \\
\hline C-reactive protein(mg/L) & $42.3(20.9-103.6)$ & $87.2(37.1-185.1)$ & 0.105 \\
\hline White blood cell(10^9/L) & $10.6(7.4-13.2)$ & $11.5(8.1-17.0)$ & 0.170
\end{tabular}

BSA: broad-spectrum antibiotics; NCBSA: nitroimidazoles combined with broad-spectrum antibiotics

Table 4 The impact of the severity of the disease on the effect of medication regimen between the two groups after propensity score matching

\begin{tabular}{llllll} 
APACHE II score & \multicolumn{2}{l}{ BSA $(\mathrm{n}=149)$} & \multicolumn{2}{l}{ NCBSA $(\mathrm{n}=149)$} & \multirow{2}{*}{$\mathrm{P}$} \\
\cline { 2 - 5 } & Effective & Invalid & Effective & Invalid & \\
\hline 0-10 score & $109(87.9 \%)$ & $15(12.1 \%)$ & $103(80.5 \%)$ & $25(19.5 \%)$ & 0.106 \\
\hline $11-15$ score & $13(65.0 \%)$ & $7(35.0 \%)$ & $13(76.5 \%)$ & $4(23.5 \% \bigotimes$ & 0.447 \\
\hline ه16 score & $3(60.0 \%)$ & $2(40.0 \%)$ & $1(25.0 \% \square$ & $3(75.0 \%)$ & 0.294 \\
\hline Total & $125(83.9 \%)$ & $24(16.1 \%)$ & $117(78.5 \%)$ & $32(21.5 \%)$ & 0.299
\end{tabular}

BSA: broad-spectrum antibiotics; NCBSA: nitroimidazoles combined with broad-spectrum antibiotics

Table 5 The impact of abdominal surgery on the outcome between the two groups after propensity score matching

\begin{tabular}{llllll}
\multirow{2}{*}{ Whether surgery } & \multicolumn{2}{l}{ BSA $(\mathrm{n}=149)$} & \multicolumn{2}{l}{ NCBSA $(\mathrm{n}=149)$} & \multirow{2}{*}{$\mathrm{P}$} \\
\cline { 2 - 5 } & Effective & Invalid & Effective & Invalid & \\
\hline Surgery & $36(94.7 \%)$ & $2(5.3 \%)$ & $73(79.3 \%)$ & $19(20.7 \%)$ & $\mathbf{0} 030$ \\
\hline Non-surgery & $89(80.2 \%)$ & $22(19.8 \% \triangle$ & $44(77.2 \%)$ & $13(22.8 \%)$ & 0.652 \\
\hline Total & $125(83.9 \%)$ & $24(16.1 \%)$ & $117(78.5 \%)$ & $32(21.5 \%)$ & 0.236
\end{tabular}

BSA: broad-spectrum antibiotics; NCBSA: nitroimidazoles combined with broad-spectrum antibiotics 
Table 6 The impact of abdominal surgery on hospitalization days between the two groups after propensity score matching

\begin{tabular}{llll} 
Whether surgery & BSA $(\mathrm{n}=149)$ & $\mathrm{NCBSA}(\mathrm{n}=149)$ & $\mathrm{P}$ \\
\hline Surgery & $18.5(10.5-43.8)$ & $33.5(20.0-51.0)$ & $\mathbf{0 . 0 0 6}$ \\
\hline Non-surgery & $18.0(9.0-33.0)$ & $17.0(10.5-32.0)$ & 0.957 \\
\hline Total & $18.0(9.5-34.0)$ & $27.0(15.0-47.5)$ & $\mathbf{0 . 0 0 1}$
\end{tabular}

BSA: broad-spectrum antibiotics; NCBSA: nitroimidazoles combined with broad-spectrum antibiotics

Table 7 The impact of abdominal surgery on expenses between the two groups after propensity score matching

\begin{tabular}{llll} 
Whether surgery & BSA $(\mathrm{n}=149)$ & NCBSA $(\mathrm{n}=149)$ & $\mathrm{P}$ \\
\hline Surgery & $117648.1(40624.4-334870.9)$ & $219313.9(124812.8-219313.9)$ & $\mathbf{0 . 0 0 8}$ \\
\hline Non-surgery & $70698.3(20592.8-207831.1)$ & $94968.7(47795.0-249144.4)$ & 0.105 \\
\hline Total & $85185.7(24028.0-313174.3)$ & $187898.3(75843.0-374426.3)$ & $\mathbf{0 . 0 0 0}$
\end{tabular}

BSA: broad-spectrum antibiotics; NCBSA: nitroimidazoles combined with broad-spectrum antibiotics

\section{Figures}




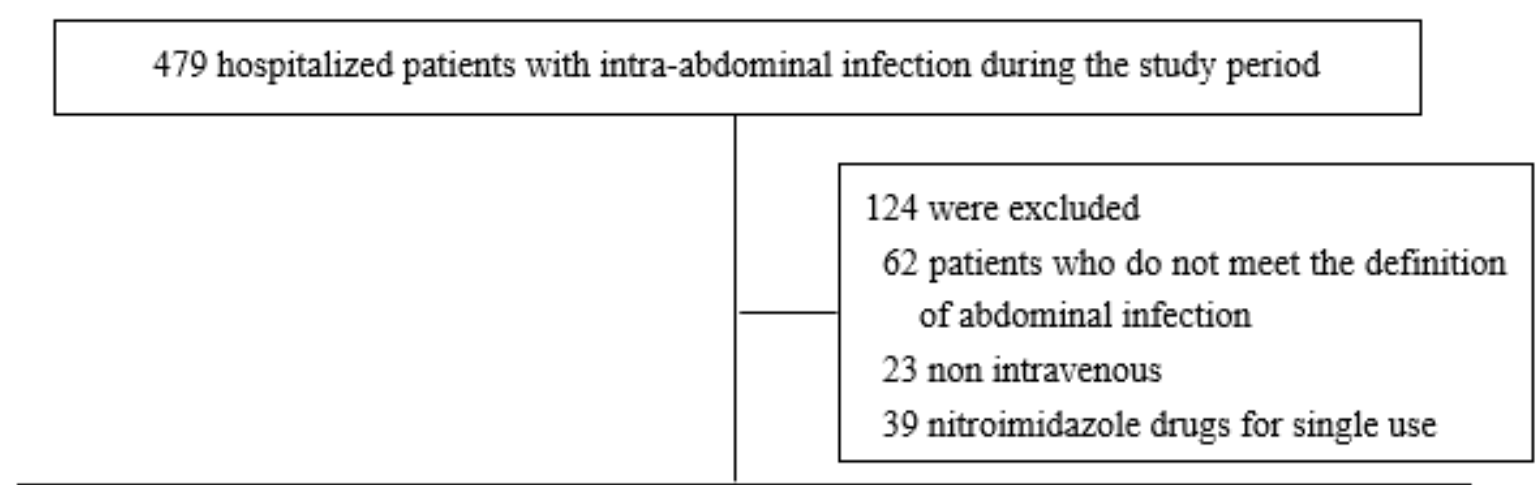

355 patients were included

(158 nitroimidazoles combined with broad-spectrum antibiotics :197 broad-spectrum antibiotics)

\section{Figure 1}

Inclusion and grouping flow chart with intra-abdominal infection patients

\section{Supplementary Files}

This is a list of supplementary files associated with this preprint. Click to download.

- Supplementaryresults20211007.docx 